\title{
Resorbable scaffold based chronic neural electrode arrays
}

Frederik Ceyssens*a, ${ }^{* a}$ ris van Kuyck ${ }^{\mathrm{b}}$, Greetje Vande Velde ${ }^{c}$, Marleen Welkenhuysen, Linda Stappers, Bart Nuttin $^{f}$ and Robert Puers ${ }^{\mathrm{g}}$

${ }^{a}$ KU Leuven, dept. ESAT-MICAS, Kasteelpark Arenberg 10, 3001 Leuven, Belgium. Email: fceyssen@esat.kuleuven.be

${ }^{b}$ Laboratory of Experimental Functional Neurosurgery, Department of Neurosciences, KU Leuven, Provisorium II, Minderbroedersstraat 19, 3000 Leuven, Belgium. Email: kris.vankuyck@med.kuleuven.be

${ }^{\mathrm{c}}$ Biomedical MRI, KU Leuven, O\&N, Herestraat 49 box 505, 3000 Leuven, Belgium. Email: greetje.vandevelde@med.kuleuven.be

${ }^{\text {d Imec vzw, Kapeldreef 75, } 3001 \text { Leuven, Belgium. welkenh@imec.be }}$

${ }^{e}$ KU Leuven, dept. MTM, Kasteelpark Arenberg 44, 3001 Leuven, Belgium. Linda.stappers@mtm.kuleuven.be

${ }^{\mathrm{f}}$ Laboratory of Experimental Functional Neurosurgery, Department of Neurosciences, KU Leuven, UZ Herestraat 49 - box 7003, 3000 Leuven, Belgium. Email: bart.nuttin@med.kuleuven.be

${ }^{g_{K} U}$ Leuven, dept. ESAT-MICAS, Kasteelpark Arenberg 10, 3001 Leuven, Belgium. Email: robert.puers@esat.kuleuven.be

*Corresponding author. Tel+3216321093, Fax +3216321975.

\begin{abstract}
We have developed a novel type of neural electrode array for future brain-machine interfaces (BMI) and neural implants requiring high resolution recording and stimulation on the surface of brain lesions or on the cortex.

The devices differ on two points from commonly used thin film electrode arrays: first, the thin film backbone of the implant is exceptionally thin (down to 5 microns) and finely patterned into springlike structures. This increases the flexibility of the electrode array and allows stretching and conforming better to a quasi spherical cavity surface. Second, the thin film backbone of the device is reinforced with a porous layer of resorbable chitosan.
\end{abstract}


This design aims at minimal invasiveness and low mechanical irritation during prolonged use, while the chitosan matrix ensures the implant is stiff enough for practical handling during the implantation procedure and dissolves afterwards.

Furthermore, the chitosan adds haemostatic and antiseptic properties to the implant and improves adhesion.

In the article, the design and fabrication process are presented. In vitro and long term in vivo test results over a twelve month period are shown. By adopting the use of a resorbable scaffold-like material as main constituent of neural implants, the presented work opens up the possibility of applying tissue engineering techniques to further improve neural implant technology.

\section{Keywords}

Neural electrode, neural electrode array, $\mathrm{BCl}, \mathrm{BMI}, \mathrm{ECoG}$, chitosan, scaffold, neural recording, neural stimulation, thin film, chronic

\section{Introduction}

Currently, neural electronic interfaces are a very active research topic worldwide. The development of high resolution, long lasting neural electronic interfaces is essential both for basic neurophysiological research and advanced therapeutic applications. In order to surpass the resolution of EEG measurements it is necessary to bring miniaturized electrodes under the skull, close to the neurons they interface with. Needle-shaped electrode arrays penetrating the cortex have potentially the highest resolution, and can e.g. record action potentials (APs) from a single neuron. Over the course of several decades, needle-shaped neural electrodes have evolved from simple insulated wires to precisely defined multi-contact silicon microneedles (Peeters et al., 1991), and further to needle beds comprising over one hundred contacts (Bai et al., 2000; Aarts et al., 2008; Blanche et al., 2005) and integrated electronics (Wise et al., 2004).

However, there is still significant concern over the lifetime of such implants which seldomly surpasses the one year mark (Ryu and Shenoy, 2009; Polikov et al., 2005; Kipke et al., 2003) due to glial scar formation. Also, stability is an issue and microneedle based brain-machine interfaces need regular recalibration (Chao et al., 2010; Chestek et al., 2007). A second, less invasive methodology is to place electrodes directly on the cortical surface (i.e. on the pia mater in humans) in a so-called electrocorticographic configuration (ECoG or $\mu \mathrm{ECOG}$ ). 
Such an approach cannot record action potentials (APs), generated by a single neuron. However, given that the highest expected spatial frequency of gamma activity of the human neocortex is between 0.4 and 0.6 cycles $/ \mathrm{mm}$ (Freeman et al., 2000), a sufficiently small electrode pitch suffices for an aliasing-free brain-machine interface based on that gamma activity. Furthermore, long term stability of the ECoG approach to brain-machine interfacing is reported to be excellent (Schalk, 2010; Chao et al., 2010), and a reduced risk of infection and bleeding is to be expected. ECoG electrode arrays boasting several hundreds of contacts (Rubehn et al., 2009; Viventi et al., 2011) have recently been reported, the latter even including active circuitry. Similar arrays are also being explored for stimulation applications, such as e.g. in retinal implants (Mokwa, 2004).

In this article, we present a prototype of a novel type of ECoG electrode array, which is especially designed for later use in hollow brain cavities as can typically be found after trauma or tumor surgery. The electrode differs on two points from common thin film electrode arrays: first, the insulating and conducting layers of the thin film backbone are finely patterned into spring-like structures, increasing the flexibility of the electrode and allowing some stretch. Furthermore, this allows the electrode to conform better to a quasi spherical cavity surface, a gyrus or sulcus of the brain. Second, the thin film structure of the electrode array is reinforced with a porous layer of resorbable chitosan which ensures sufficient stiffness for practical handling during the implantation procedure and adds haemostatic and antiseptic properties to the implant, as further detailed in section 2 below.

As further discussed in section 6, the implant can be regarded as a first instance of an instrumentalized scaffold, which could be applied to brain tissue engineering and link the field of tissue engineering with that of neural implants. Yet another perspective on the implant is to consider it an instrumentalized version of the resorbable haemostatic tissues such as Surgicel in use in surgery for many decades (Voormolen et al., 1987).

\section{Design}

\subsection{Selection of resorbable layer}

The starting objective was to design a very fine, highly flexible electrode array implant for use on the cortex or in lesions in the brain, reinforced by a resorbable material to allow practical handling during surgery. The resorbable material would dissolve once implanted, leaving a minimally invasive electrode in intimate contact with the brain (Kim et al., 2010). However, a resorbable material can do more than temporarily reinforce the implant mechanically. For the following reasons chitosan, a derivative of naturally harvested chitin obtained from it by deacetylation, was selected over more 
commonly used resorbable materials such as polylactic acid or polyglycolic acid. First, as will be shown the material is compatible with the thin film fabrication process used to produce the nonresorbable backbone of the electrode array. Unlike oxidized cellulose which is also used for resorbable haemostatic tissues such as Surgicel, it can be dissolved in an acidic aqueous environment, allowing application by e.g. spin coating. Furthermore, it can be formed into a highly porous state (Ho et al., 2004) which easily conforms to a surface when wet, and which facilitates ingrowth of cells (Crompton et al., 2006) that can anchor the implant at a fixed position on a lesion surface. At least in vitro and in subcutaneous experiments, it was shown that the degradation rate can be controlled by varying the degree of deacetylation (Lee et al., 1995; Tomihata and Ikada, 1997).

Tomihata and Ikada measured the in vivo degradation rate of non-porous $80 \%$ deacetylated chitosan to be about $1 \mu \mathrm{m}$ per day. Based on that number, a porous thin-walled structure such as fabricated in this work should resorb in a period of a few weeks.

A finally advantage of chitosan are its strong haemostatic (Brandenberg et al., 1984; Wedmore et al., 2006) and antibacterial (Kim et al., 2008) properties.

\subsection{Thin film electrode design}

In general, the electrode arrays will need to be attached to curved surfaces such as a gyrus of the brain or inside cavities. It is geometrically impossible to create intimate contact between a planar thin sheet and a spherical surface without wrinkling or excessive stress (Hure et al., 2011), which would diminish adhesion. For this reason, the arrays are not designed as a simple planar array, but are in an even more malleable, finely patterned shape. Two distinct electrode array layouts were designed, fabricated and tested: very fine 'star shaped' electrode arrays with electrodes at the end of antenna-like structures a mere 10 microns wide, and more robust 'net shaped' arrays (figure 1 ).

In a series of animal experiments similar to those using net shaped arrays described in section 5.3, the former were sometimes observed to sink in the brain. This might yield an interesting delivery mechanism for electrodes for deeper brain readout or stimulation, which is left for further work. The rest of the research described was done with the net shaped arrays, which did not sink in. 

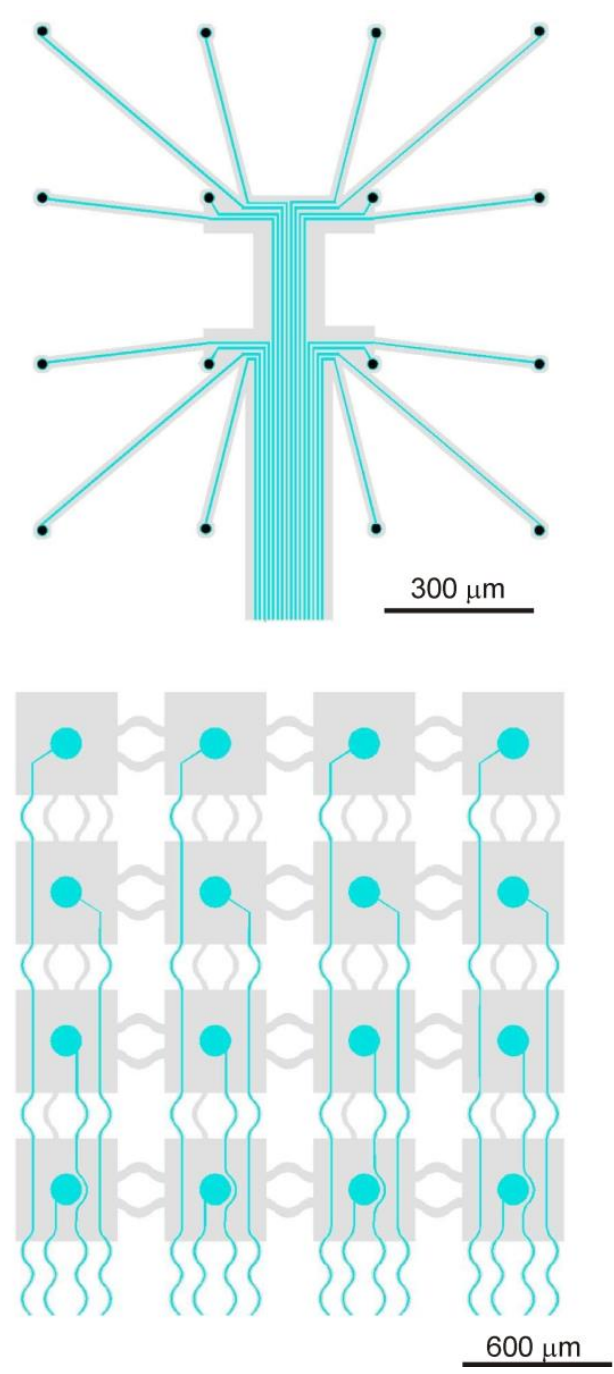

Figure 1: layout of the non-resorbable thin film part of the star shaped (top) and net shaped (bottom) electrode arrays presented in this study. Blue $=$ Pt metallisation, gray $=$ insulation layers. The lower extension containing bondpads for attaching an Omnetics connector is not shown.

The net shaped arrays (figure 1, bottom) consist of $0.4 \mathrm{~mm}$ wide square islands containing a single $\mathrm{Pt}$ electrode, connected by 200 micron long spring-like structures. This design is a compromise between flexibility and stability. Using finite element (FE) simulation (figure 2), it was proven that folding this structure around a $1 \mathrm{~cm}$ radius sphere requires $30 \%$ less energy compared to a full sheet electrode. $\mathrm{A}$ Lambert azimuthal equal area projection was assumed here. An even more compliant shape would yield even a lower bending energy. An optimization in this direction with the avoidance of sinking in as a boundary condition would be of interest but is beyond the scope of this work. 


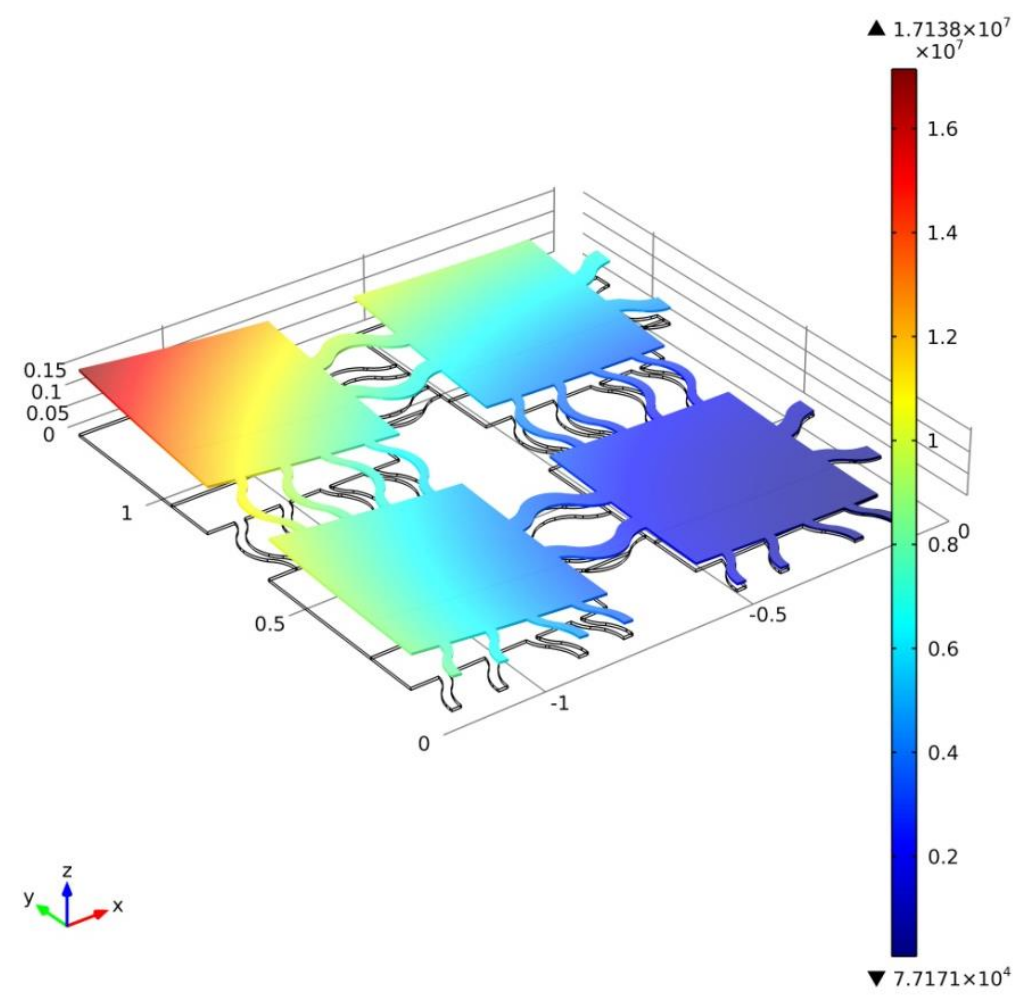

Figure 2: FE simulation of deformation energy $\left[\mathrm{J} / \mathrm{m}^{3}\right]$ required for bending a net shaped electrode array to a $1 \mathrm{~cm}$ radius. Axes are in $\mathrm{mm}$.

The electrode contacts of the net shaped array are $92 \mu \mathrm{m}$ in diameter, and allow stimulation next to ECoG monitoring. Using an electrochemically and histologically safe current density limit of 30 $\mu \mathrm{C} /$ phase $/ \mathrm{cm}^{2}$ of real surface area (van Kuyck et al., 2007) and $0.06 \mathrm{~ms}$ long biphasic pulses a stimulation current of $33 \mu \mathrm{A}$ can be injected safely. The electrodes are placed at a pitch of 675 micrometer which surpasses the requirements of (Freeman et al., 2000) and places an electrode over each cortical column (Mountcastle, 1997). Moreover, the fabrication process elaborated below allows to place electrodes on the upper as well as on the lower side of the array.

The thin film electrode arrays also contain bondpads, designed for attaching an Omnetics Nano Strip 18-pin connector, model number NPD-18-VV-GS. These are located $4 \mathrm{~mm}$ from the nearest electrode.

\section{Fabrication}

First, the thin film backbone of the design is fabricated using lithography on silicon carrier wafers. In the final steps of the fabrication procedure, the resorbable porous chitosan is applied and the complete electrode array is released from the silicon surface. The more detailed outline of the fabrication procedure (illustrated in the top part of figure 3 ) is as follows: 
1. Standard silicon wafers are cleaned by a piranha $\left(4 \mathrm{H}_{2} \mathrm{SO}_{4}: 1 \mathrm{H}_{2} \mathrm{O}_{2}\right)$ clean, a diluted $\mathrm{HF}$ dip and a 5 minute rinse in an ultrasonic bath with streaming DI water.

(1)

(2)

(3)

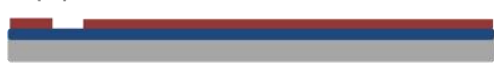

(4)

(5)

(6)

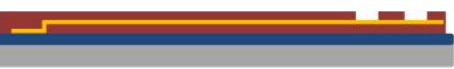

(7)

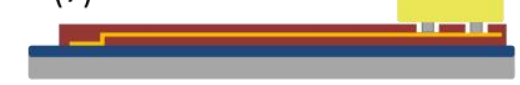

(8)

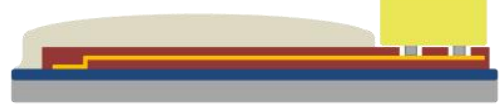

(9)

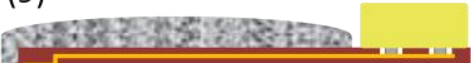

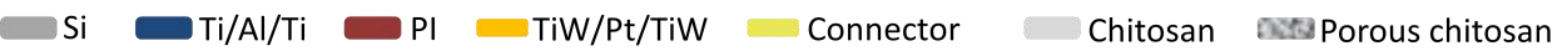

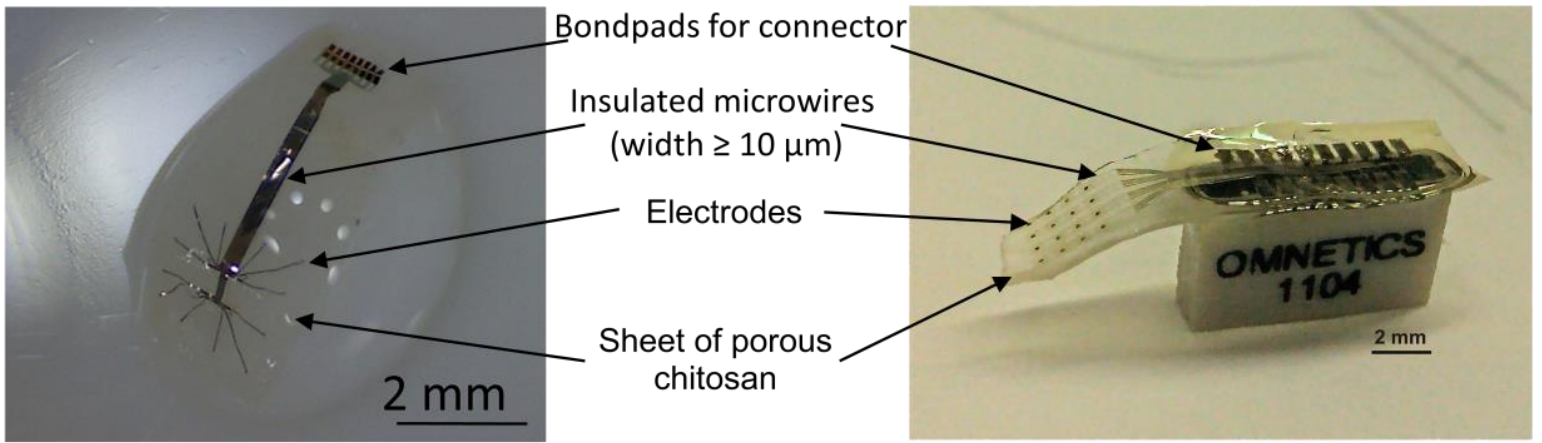

Figure 3: Top: fabrication sequence. Lower left: fabricated 'star shaped' electrode array with chitosan reinforcing matrix, floating on a droplet of saline water. Lower right: fabricated net shaped electrode array with porous chitosan reinforcing matrix, mounted on an Omnetics connector.

2. A Ti-Al-Ti stack is deposited (thickness $50 / 250 / 25 \mathrm{~nm}$ ) to serve as a sacrificial layer. The Ti and Al form a galvanic couple similar to (Metz et al., 2005; Aarts et al., 2011), speeding up later release steps. During later wet processing steps, the top titanium serves to prevent undercut of openings etched in the subsequently deposited insulation layer. This would create a discontinuity in metal layers connecting the top and bottom of the openings.

3. In order to produce the first insulation layer, diluted PI2611 polyimide $(0.2857 \mathrm{~g} \mathrm{~N}$-Methyl-2pyrrolidone (NMP) added per gram of PI 2611) is spin coated at $2000 \mathrm{rpm}$ and hardened according to the protocol in the datasheet, yielding a 3 micrometer thick layer. To create electrode contact openings, the layer is etched using a 5 micron thick positive S1818 photoresist mask in a reactive ion etcher (RIE, etch parameters: 100W power, 100 mtorr pressure, $40 \mathrm{sccm}$ 02, $20 \mathrm{sccm}$ SF6, $11 \mathrm{~min}$.). 


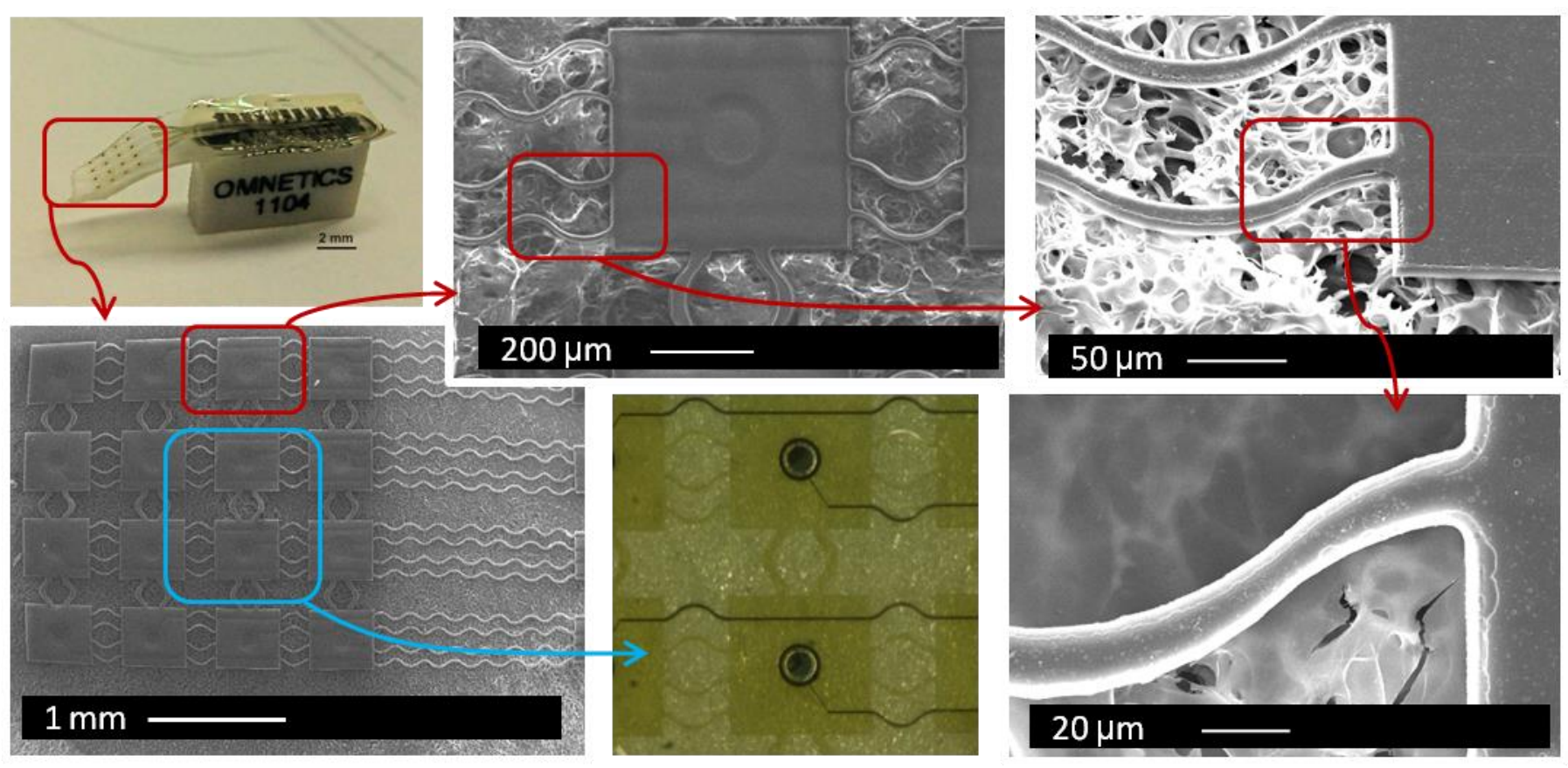

Figure 4: SEM pictures of 'net shaped' electrode array, reinforced with porous chitosan. Note that these are taken in a dry (i.e. shrunken) state. Based on macroscopic shrinking, pore size when wet should be about $50 \%$ larger.

4. A liftoff resist bilayer is deposited (LOR1OB and S1818 photoresist) and lithographically patterned. Then, the polyimide is activated by sputter etching ( 6 inch chuck diameter, $1^{\prime} 50 \mathrm{~W}, 3.10^{-3} \mathrm{mbar} \mathrm{Ar}$ ) and a TiW-Pt-TiW stack is sputter deposited $(10 / 160 / 10 \mathrm{~nm})$ immediately afterwards. The liftoff process is completed by soaking in NMP overnight, and a 1 minute step in fresh NMP in an ultrasonic bath.

5. After a 45 second plasma activation step ( 02 plasma, 50W, 150 mtorr) to increase adhesion, the second insulation layer is deposited using the same parameters as for the first.

6. An $80 \mathrm{~nm}$ thick aluminum layer is deposited by evaporation to serve as etch mask and is lithographically patterned. A second RIE is used to define the contours of the electrode arrays. As Pt is inert to the RIE plasma, the same etch will stop on the Pt bond pads and open them up: for this reason, the openings in the etch mask overlap $10 \mathrm{~mm}$ with the bond pads. Then, the aluminium is removed chemically in $\mathrm{NaOH}$.

7. The carrier wafer is diced and the Omnetics connector is mounted on the bond pads. Then, the bond pads are isolated using a biocompatible epoxy-based underfill (Epo-Tek 302-3M). Adhesion of this underfill is improved by plasma activation as in step 5 . 
8. High molecular weight ( $\mathrm{Mw}$ ) chitosan (75-85\% deacetylated, ) was purchased (Sigma-Aldrich nb. 419419), dissolved to $2 \mathrm{Wt} \%$ in $1 \mathrm{M}$ acetic acid and centrifuged at $4566 \mathrm{~g}$ for 15 minutes to remove particles. A layer of the solution is applied by casting and spreading with a blade held $0.5 \mathrm{~mm}$ above the substrate.

9. A freeze-gelation method similar to (Ho et al., 2004) is used to create the porous chitosan layer: the carrier substrate is frozen to $-20^{\circ} \mathrm{C}$ and then put into a $1 \mathrm{M} \mathrm{NaOH}$-ethanol solution pre-cooled to the same temperature for 12 hours. As the solubility of chitosan is lower in frozen water and the material does not dissolve in an alkaline environment, this step solidifies the chitosan into a porous layer. Furthermore, it releases the electrode array from its substrate by dissolving the aluminum sacrificial layer in the same step. The exposed copper parts of the connector are not damaged by this step. Afterwards, the arrays are rinsed in ethanol. A dip in diluted HF to remove the thin Ti layer attached to the lower side of the probe concludes the fabrication procedure.

After fabrication, the implants are kept in $70 \%$ isopropanol for desinfection purposes. Before implantation, they are rinsed three times in sterile isotonic saline. The implants are implanted while still moistened with saline in order to avoid shrinkage.

Figure 4 shows more detailed scanning electron microscope (SEM) pictures of the fabricated net shaped electrodes. A good adhesion between the chitosan and the thin film layer was achieved. Down to $10 \mu \mathrm{m}$ wide, up to several millimeter long freestanding 'antenna shaped' electrodes, consisting of a platinum conductive layer surrounded by dielectric on all sides, could be reliably fabricated with this process. A variant of this process using a 2.5 micron thick Parylene $C$ layer as insulation instead of polyimide was shown to work as well. However, these electrodes were not ready in time for the in vivo experiments.

\section{Impedance characterization}

In vitro testing of electrode impedance was done in phosphate buffered saline water (Invitrogen Gibco, measured $\mathrm{pH} 7.1+/-0.1$ ) using a platinum counter electrode and a $\mathrm{Ag} / \mathrm{AgCl}$ reference electrode. A Solartron SI 1255 frequency response analyser and a SI 1287 electrochemical interface were used to measure impedance of 12 electrodes in a range between $0.1 \mathrm{~Hz}$ and $1 \mathrm{MHz}$. A $0.1 \mathrm{~V}$ sinusoidal waveform was used. The measured impedances are shown in figure 5, together with a simple equivalent circuit model fit to the data.

They are well below the input impedance of the amplifiers used for in the in vivo tests (16 M $\Omega$ at 1 $\mathrm{KHz}$ ), and thus acceptable for applications in physiological measurements. 
Furthermore cyclic voltammograms of the electrodes were measured in PBS solution as shown in figure 6. A scan rate of $100 \mathrm{mV} / \mathrm{second}$ was used, and three consecutive cycles were measured. Hydrogen and oxygen evolution respectively start at potentials of -0.6 and 1.1 volts vs. $\mathrm{Ag} / \mathrm{AgCl}$, which is consistent with measurements on platinum electrodes published earlier [Hudak 2010, Musa 2009]. The large current step around zero volts vs. $\mathrm{Ag} / \mathrm{AgCl}$ originates from the reduction of dissolved oxygen present in the solution (Musa 2011). If remaining titanium or tungsten would be present, the hydrogen overpotential would be at least $0.3 \mathrm{~V}$ lower (Sharma, 1997).

The insulation resistance was characterized as well. After mounting the Omnetics connector and release of the electrode array from the substrate, the insulation resistance between different electrodes was determined using a Keithley $2612 \mathrm{~A}$ source meter, set to apply a maximum voltage of 20 V. An average resistance of $10.5 \mathrm{G} \Omega$ (range: 8-13 $\mathrm{G} \Omega$ ) was found."
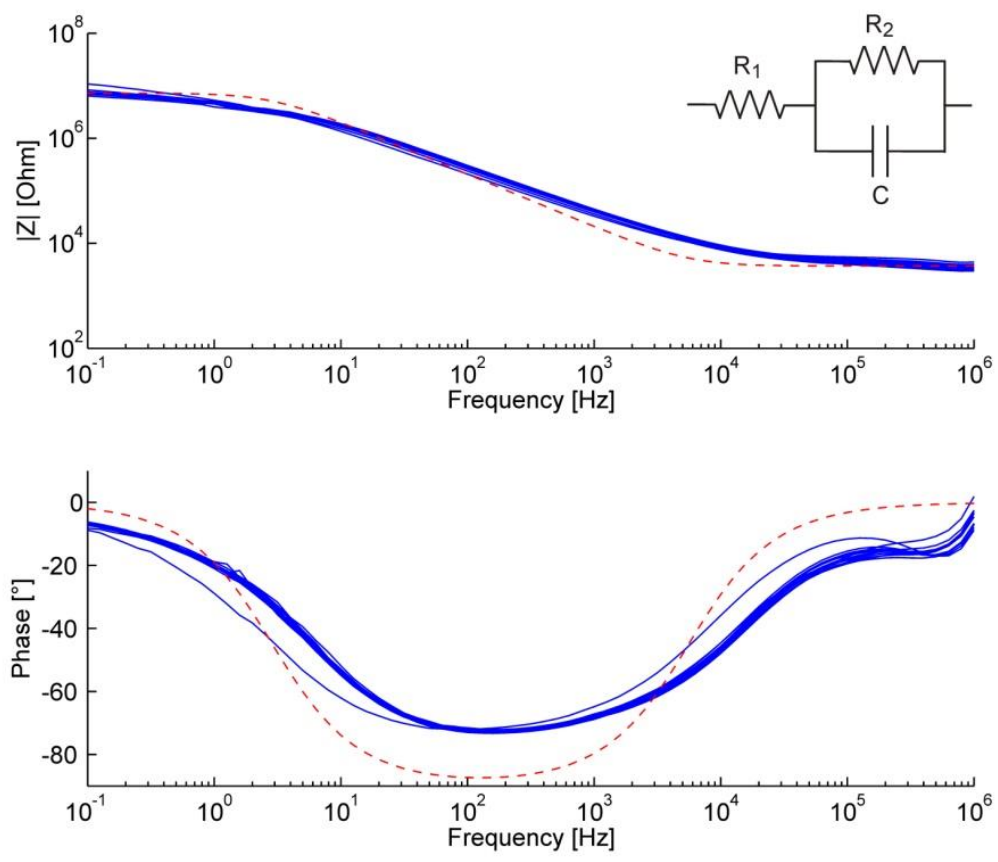

Figure 5: Absolute value and phase of the measured impedance of the electrodes of a net shaped electrode array, measured in PBS solution ( $\mathrm{pH}$ 7.1). The red dashed line displays the fit of the data to the simple equivalent circuit model displayed in the upper right corner, with $R 1=7.2 \mathrm{M} \Omega, R 2=3700 \Omega$ and $C=7.7 \mathrm{nF}$. 


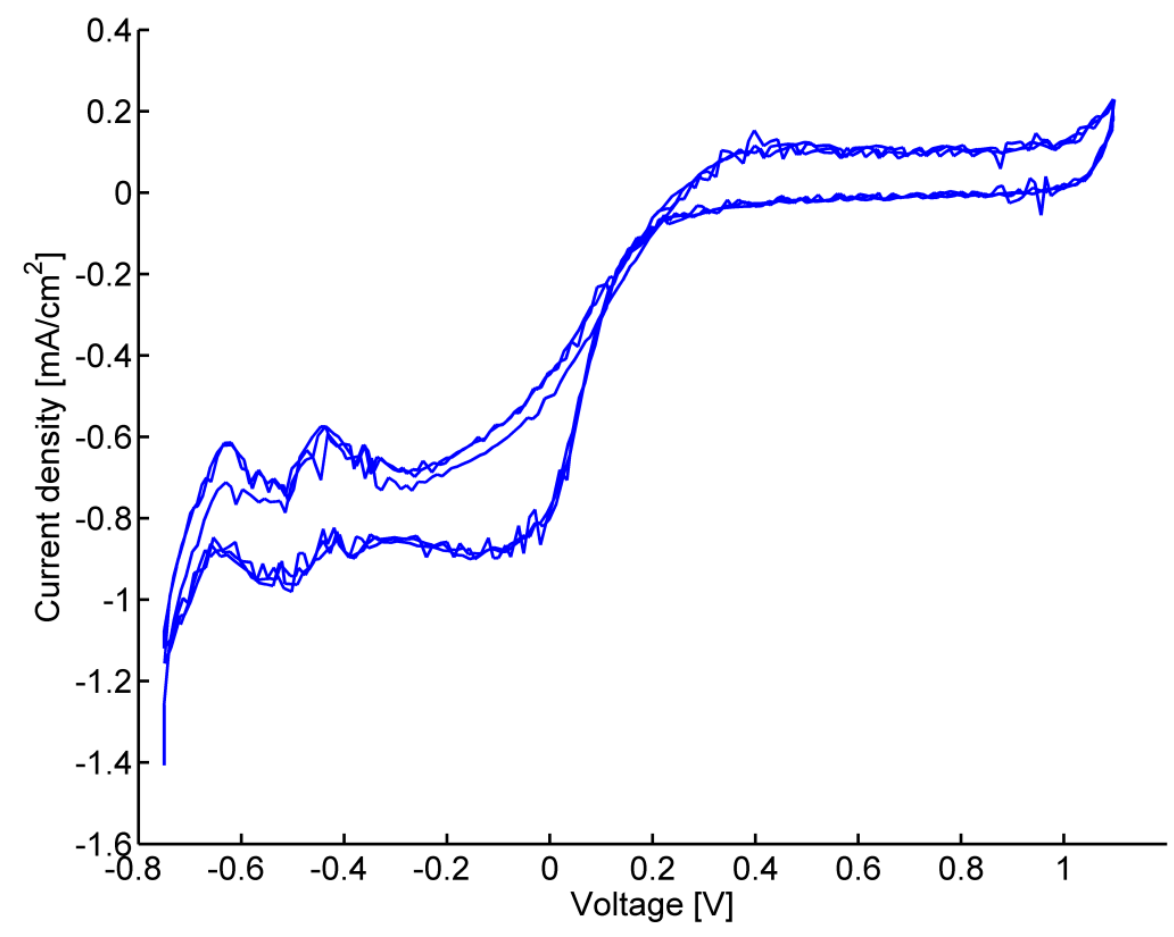

Figure 6: cyclic voltammogram of a single electrode of the fabricated arrays, showing hydrogen evolution around $-0.6 \mathrm{~V}$ and oxygen evolution around $1.1 \mathrm{~V}$. Other measured electrodes displayed a similar curve.

\section{In vivo testing}

\subsection{Experimental setup}

In order to attain an assessment of the long-term performance of the electrode arrays fabricated, a twelve month test was done in four Wistar rats codenamed MC4, 5, 10 and 11 . All experiments were carried out in accordance with protocols approved by the local university animal ethics committee and in accordance with the European Communities Council Directive of November 24, 1986 (86/609/EEC).

The net shaped electrode arrays decribed above are used. Wistar rats (200-300 g body weight) were anaesthetised using intraperitonial ketamine (Anesketin, Eurovet, Belgium) and medetomidine (Domitor, Orion Cooperation, Finland) and positioned in a stereotactic frame. The skin was opened near the sensorimotor cortex and a $3 \times 3 \mathrm{~mm}$ burr hole was drilled in the skull. The brain's meninges, which are very thin in rats, were removed in MC10 and MC11 and left in place in the other rats. Screws were put in the skull at a distance of about $1 \mathrm{~cm}$ from the opening to aid in fixating the connector and to serve as reference electrode (figure 7). The opening in the skull was closed using 
UV hardened dental cement, which was also used to fixate the Omnetics connector of the electrode array between the screws.

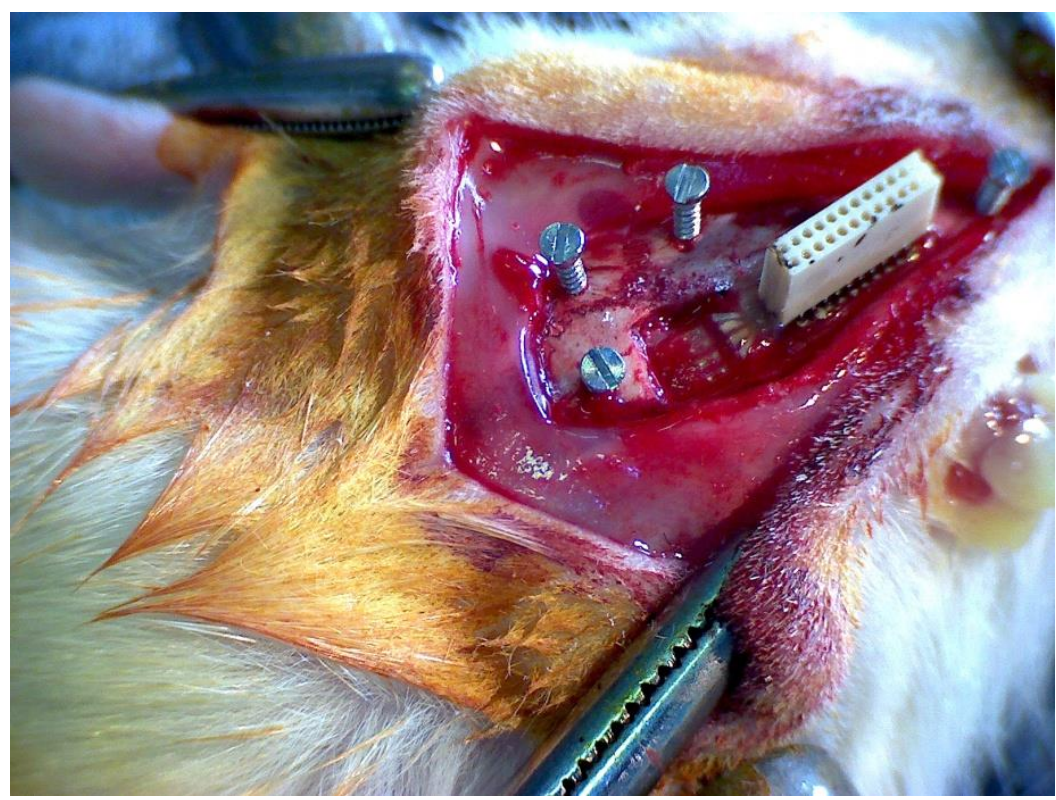

Figure 7: implantation procedure.

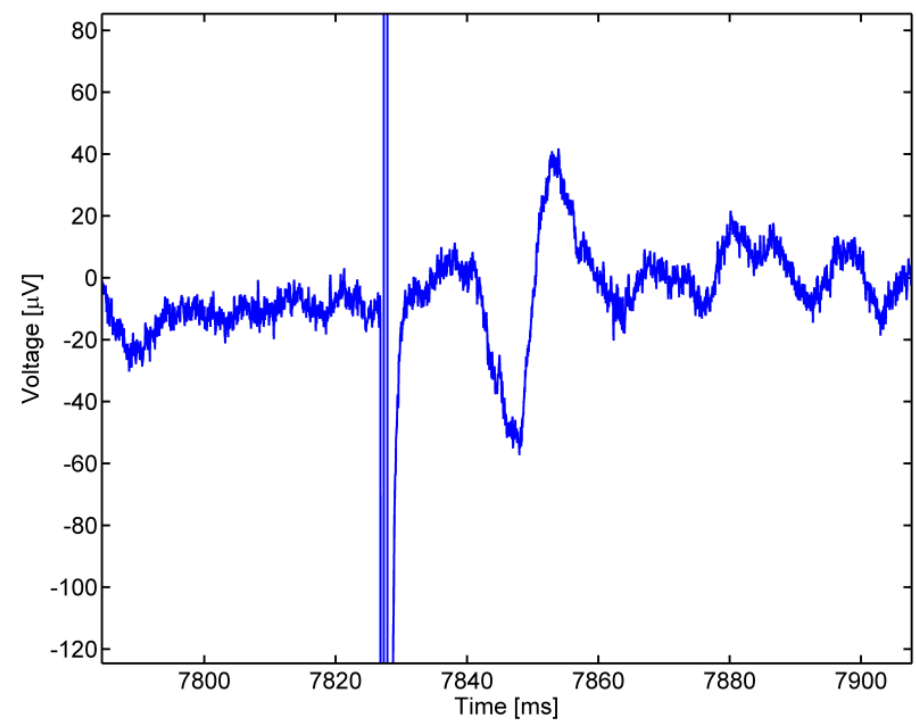

Figure 8: typical physiological reaction to stimulus in limb, showing the stimulation artifact (large peak, several $\mathrm{mV}$ ) and the physiological response $10 \mathrm{~ms}$ later.

After surgery, a platinum electrode was inserted in the back of the left forepaw and a biphasic stimulation routine was applied (biphasic pulses, 1 ms per phase wide, repeated every second for 30 
seconds, $800 \mu \mathrm{A}$ current with an anal electrode as reference). The stimulation routine was repeated after a 30 second resting period. The stimulation peak (i.e. stimulation artifact) and the physiological reaction (evoked potentials) were recorded using a Multichannel Systems USB-ME32-FAI recording system connected to the 16 channels of the electrode array.

Measurements of the reaction on an identical stimulation protocol applied to the other limbs of the test animal were made as well. Before further processing the recorded signal was high pass filtered using a finite impulse response filter (Kaisar window, $30 \mathrm{~dB}$ attenuation in stopband from 0 to $14 \mathrm{~Hz}$ ).

When averaged signals were studied, a peak detection algorithm was used that detects stimulation peaks based on a large deviation from the average signal. A threshold of a factor 6.5 above the standard deviation of the signal in the channel was employed. The signals in the intervals between 10 and $80 \mathrm{~ms}$ after each detected peak are then taken and averaged.

The measurements were reiterated seven times over the test period. Before every reiteration, the rats were anaesthetized as well.

\subsection{In vivo measurement results}

The stimulation peak could be seen in every one of the 16 channels of each electrode array during the six month course of the experiment, indicating the integrity of the electrodes over time despite the free movement of the rats in between measurements. However, the integrity of the brain tissue neighboring the implant is important as well. The latter can be assessed based on the measured physiological reaction to the stimulation peak. A typical physiological reaction is shown in figure 8 , and occurs between 10 and 30 ms after the stimulation peak. Similar reactions are also reported in literature (Hosp et al., 2008).

This signal, generated by living tissue, is inherently stochastic. Therefore, the average reaction to the stimulus was studied. In MC4 and MC5 a typical physiological response could be recorded in every channel during stimulation of at least one $\operatorname{limb}$, up until the last day of experimentation at six months. This shows good long term tolerance of the implant by the test animal. The animals were only sacrificed after an additional six months, before which the experiment was repeated once more. In MC4 the physiological reaction was no more present and had been replaced by a constant epilepsy-like pattern about $100 \mu \mathrm{V}$ in amplitude. in MC5 however, a healthy response could still be measured. There was also a correlated difference in the histology (section 5.3).

Figures 9a and 9b show the measured average response in the 16 channels after 6 and 12 months, respectively. The physiological response averaged over the 16 channels as a function of time is shown in figure 10. With the exception of a few cases in the middle of the experiment, the four 
stimulation cases could be distinguished based on this average alone. Thus, in distinguishing these rather coarsely separated inputs the electrode pitch can be significantly larger without compromising performance. In figure 10, there is a clearly dip in the observed average peak-to-peak response around day 20. This was observed in all animals. A possible explanation could be an effect of the acute immune response which is, based on the accumulation of macrophages and microglia, at its peak in that period (Lotfi 2007).
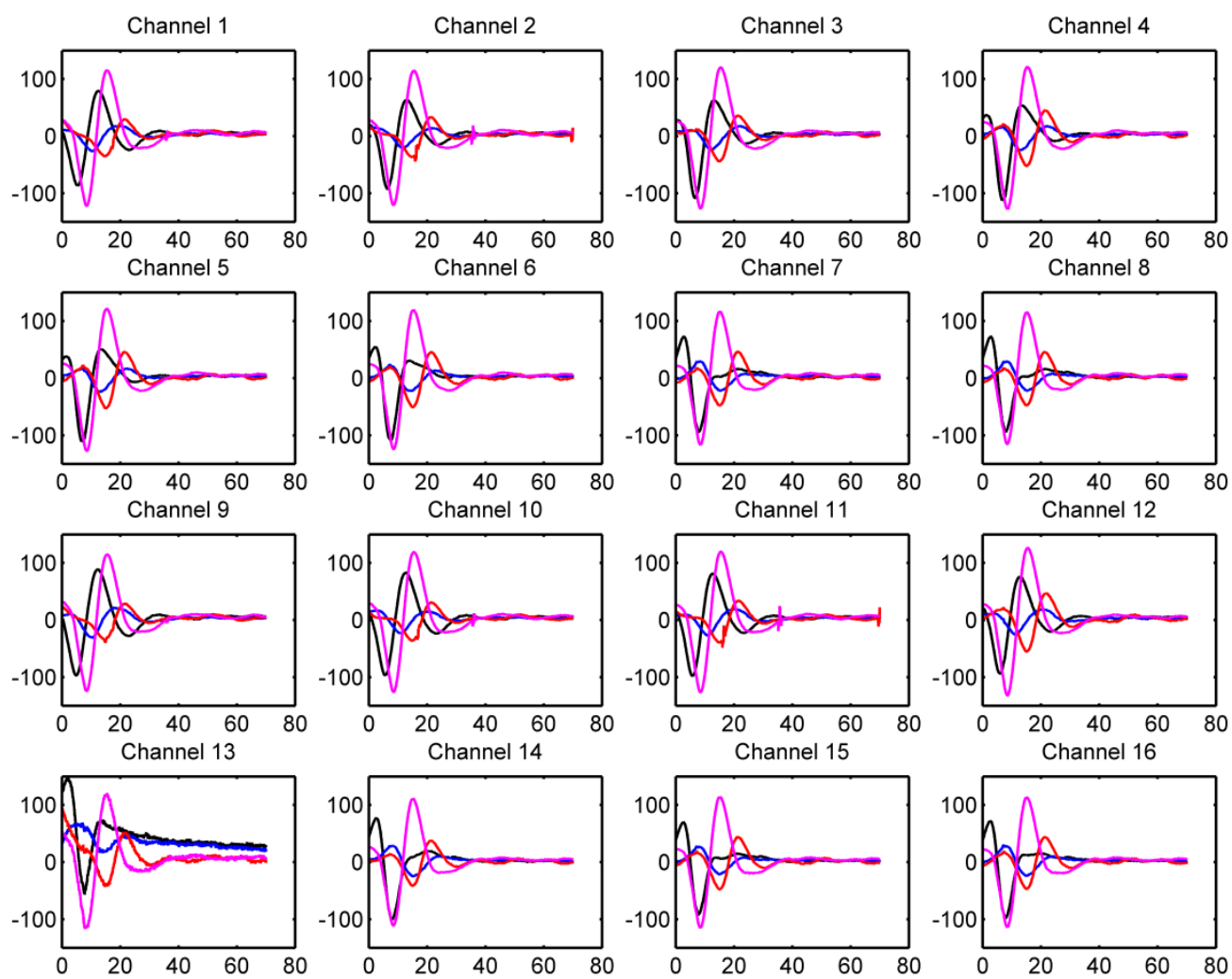

Figure 9a: Average physiological reaction to the stimulus measured by each of the 16 channels of the electrode array. Measurement taken 6 months after surgery in rat MC5. The four colors indicate stimulation of the different limbs of the test animal. Unit of $x$-axis: ms. Unit of $y$-axis: $\mu V$. Color code: left forelimb: black. Left hindlimb: magenta. Right forelimb: blue. Right hindlimb: red. 

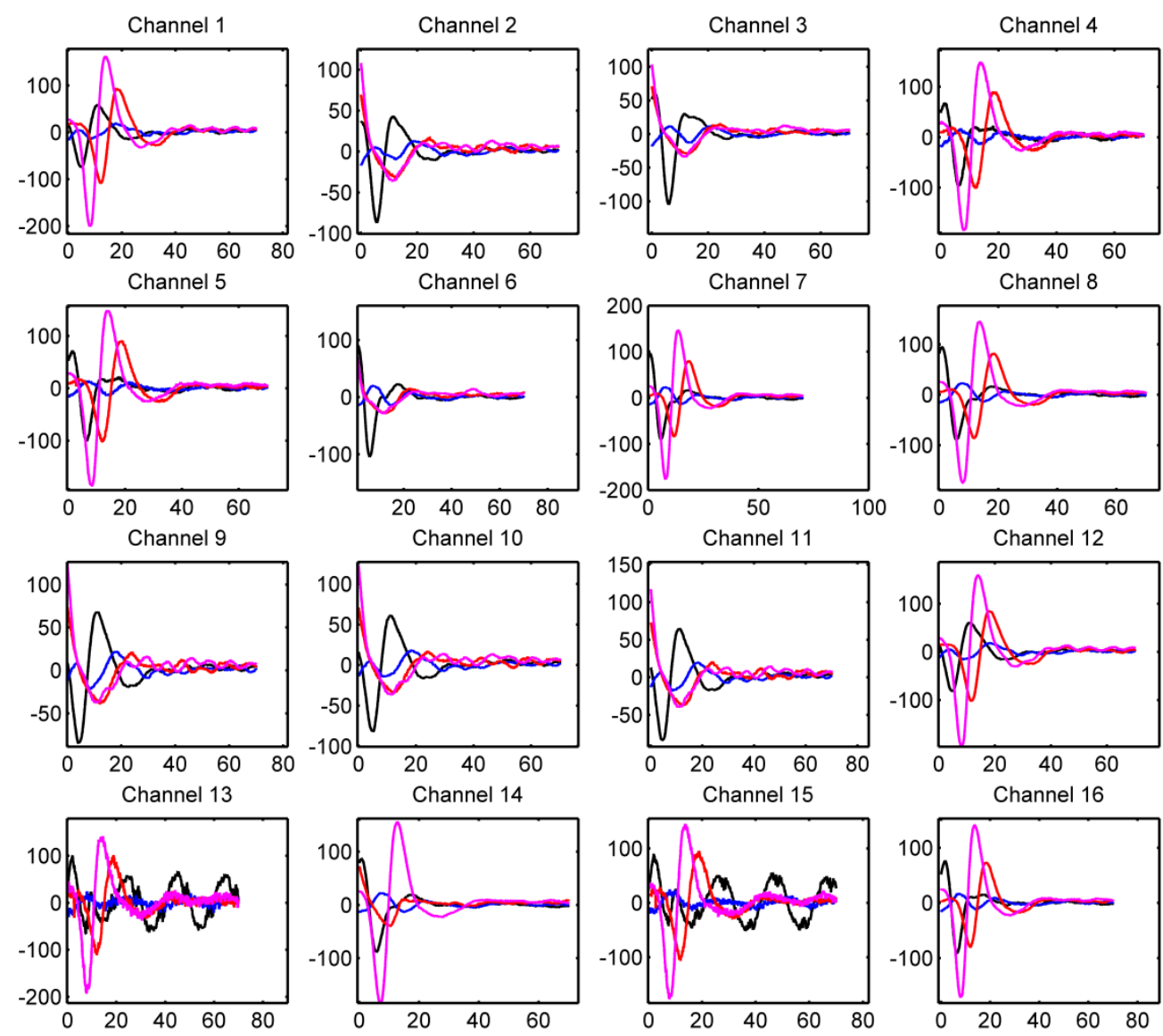

Figure 9b: Average physiological reaction to the stimulus measured by each of the 16 channels of the electrode array. Measurement taken 12 months after surgery in rat MC5.

In MC10, the dura was removed before the implant was put in place. As further shown in part 5.3 even the surgical removal of the dura alone causes significant tissue damage. This can also be seen in the recordings: in MC10 a typical physiological reaction could only be recorded on all channels at the day of the implant. From the next measurements one week later to the end of the experiment, only in $25 \%$ of the channels a physiological reaction could be seen. For MC11, no electrical measurements could be done due to a clogged connector.

\subsection{Histology and CT tracking}

A further test to assess scar tissue generation by the implant when positioned under the meninges was performed. Four dummy net electrode arrays (i.e. without connector) were implanted on the brain with the meninges removed using the same procedure as above, except no dental cement was used. In another three instances, only a chitosan tissue (fabricated using the same procedure as above) was implanted. In three other instances Surgicel, a resorbable oxidised cellulose-based 
haemostatic commonly used in brain surgery, was used. In a control group, the meninges were removed without implanting anything.

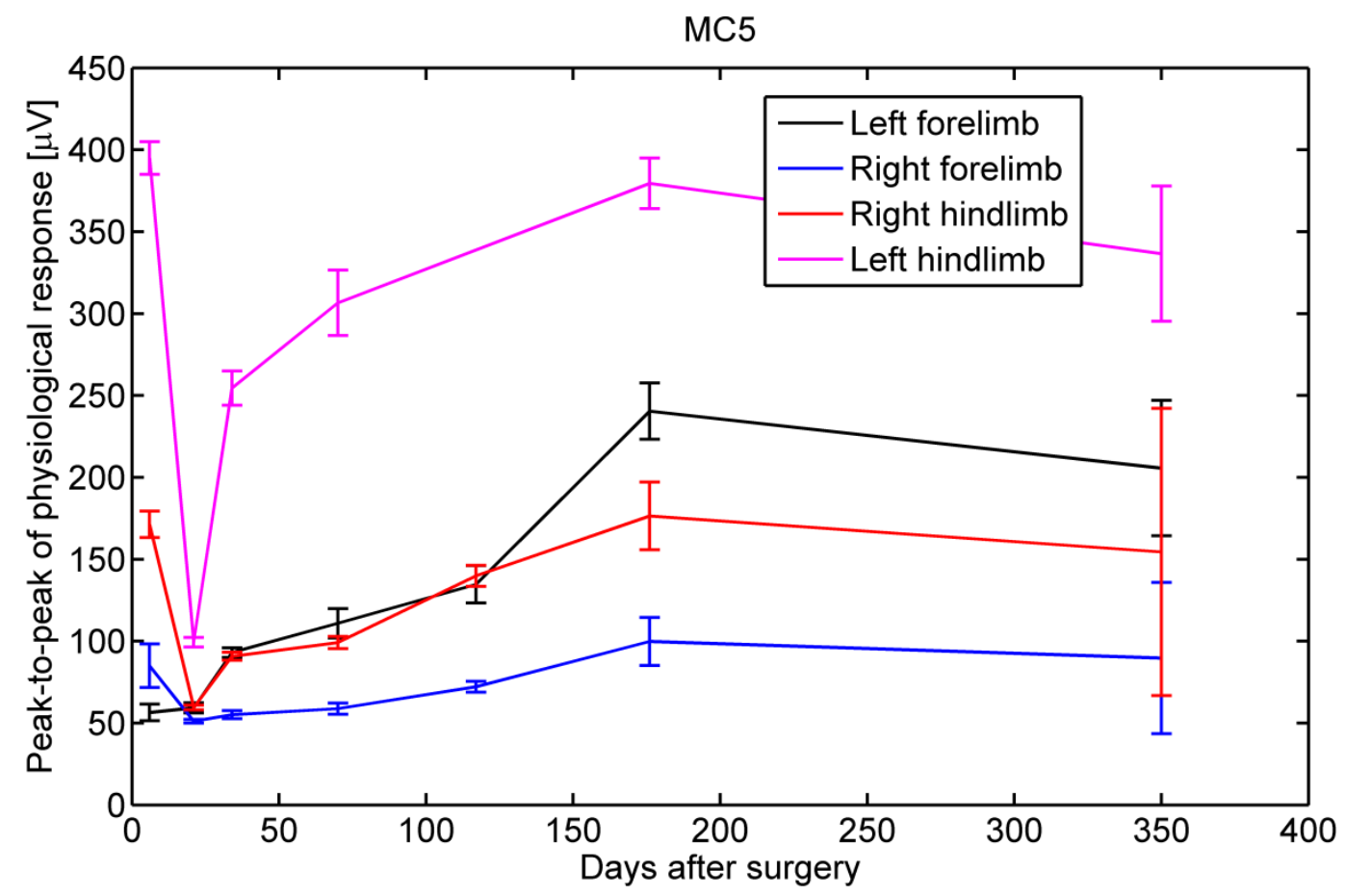

Figure 10: peak-to-peak response of MC5 averaged out over the 16 channels as a function of time after surgery, for the four stimulation cases. Error bars denote +/- one standard deviation.

Rats were sacrificed after three months, when tissue reaction is fully developed. Histological analysis (NeuN and GFAP immunostaining) was done and the depth of the scar was measured, defined as the distance between viable neurons made visible by NeuN staining in the scar and normal brain surface, measured in the section where damage is highest. The results are summarized in figure 11. Histology pictures can be found in the supplementary data. A complication with histology was the scar tissue the electrode was embedded in was only very loosely connected to the brain, and much more to the skull out of which it was hard to remove and process into slices due to the nearby bone and, in case of non-dummy implants, screws, connectors and dental cement. That is why most pictures show a cavity where the electrode or dummy electrode and the surrounding scar tissue were, and the depth of the cavity is given to quantify tissue damage.

The average depth below which viable neurons could be seen was about 420 microns in case of the full implants and 350 microns in case of the chitosan or Surgicel alone. The variation in case of 
Surgicel, which is manufactured under strict pharmacological rules, is as can be expected much lower.

A control with intact dura showed little or no damage. Therefore, it can be stated that the surgery including removal of the dura and/or the subsequent contact with non-brain tissue triggers at least as much damage as the implant itself over a 3 month period.

The amount of tissue damage observed is comparable with the damage seen after resection of longterm neural implants in human patients (Haberler et al., 2000; Henderson et al., 2004).

Furthermore, the 'net shaped' dummy arrays can also be tracked non-invasively by CT scan as was demonstrated in one live rat. In images taken using a Skyscan 1076 high resolution in-vivo CT scanner and rendered using CTvox 2.3 software, the $4 \times 4$ array of platinum contacts was still visible. Three scans were made, one week, three weeks and two months after surgery. Using this method (pictures: figure 12), the dummy net array could be seen to move about 400 microns downwards, following the contours of the retreating neural tissue.

Finally, after one year of experimentation MC4 and MC5 were sacrificed as well and a similar damage assessment by histology was done. The results are shown in figure 13. It can be seen that one rat has brain damage along the lines of the previous three-month experiment while in the others increased astrocyte counts and brain damage can be seen, which correlates with the loss of good recordings in MC4 after 6 months. A cross-section of scar tissue containing the electrode is also given in figure 13.

\section{Discussion}

We have demonstrated that thin film microelectrode arrays can be fabricated reliably into shapes much finer than currently used: freestanding $\mathrm{mm}$-long antenna shaped electrodes down to $10 \mu \mathrm{m}$ wide, consisting of a platinum conductive layer surrounded by dielectric on all sides, could be reliably fabricated with the process presented in this paper, based on contact lithography. The patterning of the thin film layer in order to improve foldability over three-dimensional curved surfaces was recently presented already in (Kim D 2010), but the achieved minimum width here is one order of magnitude finer. Furthermore, the process presented allows for top as well as bottom contact openings. 


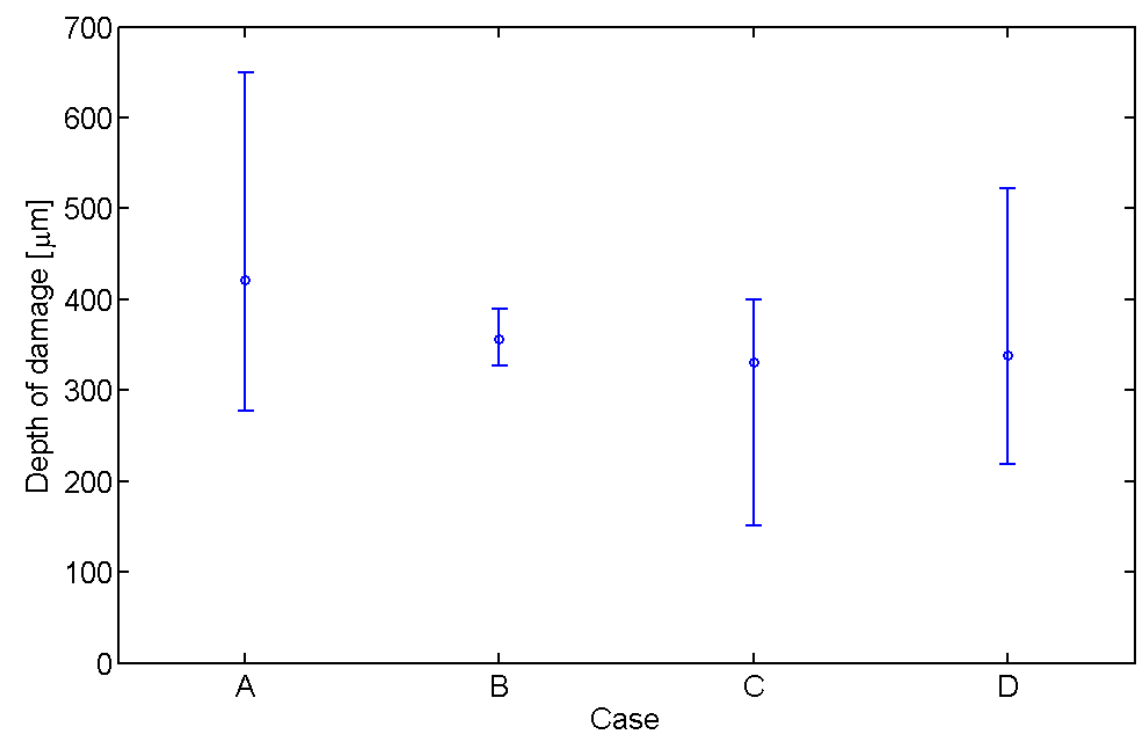

Figure 11: Histology data: depth of tissue damage 3 months after implantation in case of (A) full dummy implant (B) resorbable material (Surgicel) only (C) resorbable material (chitosan) only $(D)$ no implant, only burr hole. Average, minimum and maximum values shown. Histology pictures can be found in the supplementary data.

Also, the demonstrated fabrication process allows a matrix of resorbable, porous chitosan to be fabricated and to be well attached to the thin film part. As such, the electrode arrays fabricated can still be handled practically during an implantation procedure.

The concept is not limited to neural electrodes: the temporarily reinforced electrodes could be used in other tissues as well, for example in implants performing low-irritation electrocardiogram or electromyogram recording.

The electrode arrays have been tested on Wistar rats. It was shown that the electrode arrays can record physiological signals on all channels over a period of up to twelve months when placed on the dura.

In case a direct contact was made with the brain a physiological response could be still be detected in $25 \%$ of the channels. Still, the tissue damage induced by the implant in this case is not significantly higher than that in a control group with a removed dura, but without implant. Though we consider these results encouraging for this proof-of-concept study, as the final goal of the implant is to be used in neural prostheses in cavities in the brain this calls for further research and optimization. 


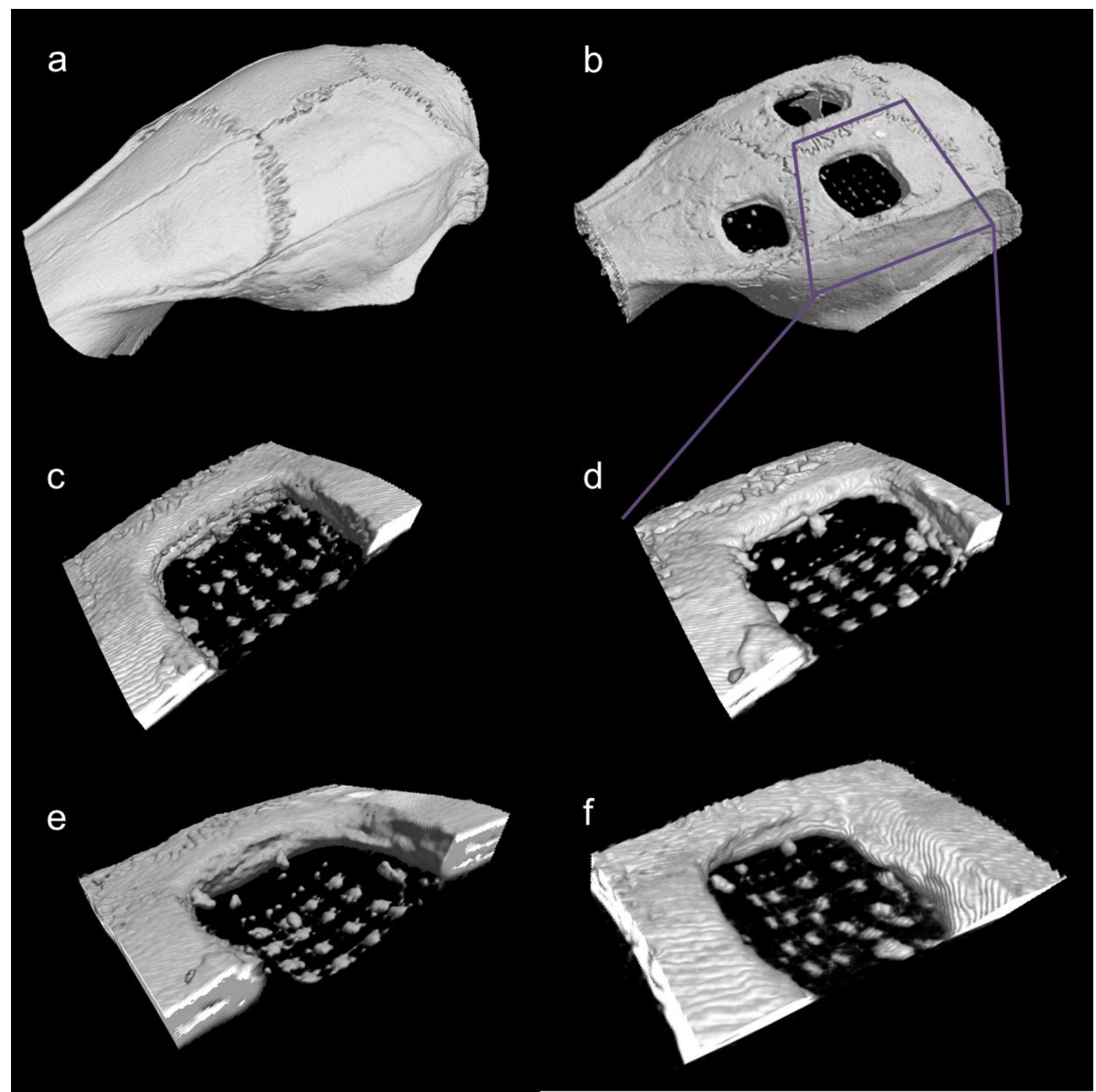

Figure 12: Longitudinal in vivo CT images of one representative rat (FC7) with implanted 'Net type' electrodes before surgery (a) and at one day (c), 1 week (b, d), 3 weeks (e) and 2 months (f) after surgery. The electrode was implanted in one burr hole (see inset in (b)). Dummy electrodes (without connector) were used. The CT images show that the electrode stays closely associated with the soft tissue contours of the rat brain and stability of this electrode position over time.

A potential issue hindering future application could be the use of toxic chemicals during fabrication, in particular HF. Though in vivo tests (section 5) show no signs of poisoning or increased tissue damage after the fabrication and rinse steps of the fabrication process presented above, in further work the risk of tissue damage by the presence of trace amounts of HF can be further reduced adding an additional rinse in an alkaline buffer after the HF dip, converting HF into relatively harmless NaF. Alternatively, the need for a top Ti layer could be avoided by using a tetramethyl ammonium hydroxide (TMAH) based developer, which has lower reactivity with aluminum. 
Being a proof-of-concept, this study did not include an effort to optimize the scaffold for optimal acceptance by the body. It was concluded from the three month histological study (section 5.3) that the surgical procedure itself was the major source of tissue damage. Thus, at first this procedure should be improved. Nevertheless, for chronic recording at a certain point the electrode itself is expected to become the dominant source of damage. The loss of physiological signal in rat MC4 between 6 en 12 months after starting the experiment can hardly be attributed to the surgery. Therefore, a long term follow-up study aimed towards optimization of the biological acceptance will be equally important.

This optimization study should include a control group with a thin-film electrode without chitosan or another scaffold as well, and of course be run on a significantly larger population. The scaffolds materials tested do not have to be limited to different types of chitosan: in further research various pharmacological substances such as anti-inflammatory agents could be tested to further improve acceptance by the body (Kim and Martin, 2006; Zhong and Bellamkonda, 2005). Also, the use of other tools such as covering the implant with extracellular matrix components (Cui et al., 2001) (e.g. laminin or fibronectin), the use of other porous resorbable materials from tissue engineering research and the application of growth factors could be attempted. The ultimate goal would be to optimize the scaffold such that neurons instead of scar tissue grow into the resorbable matrix, and optimal contact with the electrodes is established.

The electrode arrays could be used in this research to obtain 'live' data of the performance of the implanted array and the condition of the surrounding neural tissue, such as demonstrated by the dip at 20 days in figure 10 .

This brings us to the broader perspective on this work. To the authors' knowledge, for the first time a porous, scaffold-like resorbable material was used as main constituent of a neuroelectronic implant. Further exploration of this novel concept, in which the fields of tissue engineering and neural implants converge, can yield significant progress.

As already hinted above, various concepts and techniques from tissue engineering research could be applied, building on the concept presented and leading to neural implants having a more intimate relationship with the neurons they interface with. On the other hand, tissue engineering research could benefit from the added control and monitoring capabilities instrumentalized porous scaffolds such as shown in this work offer. For example, the live electrophysiological data generated could be used to monitor in vivo tissue regeneration experiments in the brain or other organs, supplementing histological data which can only be gathered at the end of an experiment. 
Further down the road, the live data generated by the system could be used to monitor and steer tissue regeneration treatment by electrical or pharmacological means.
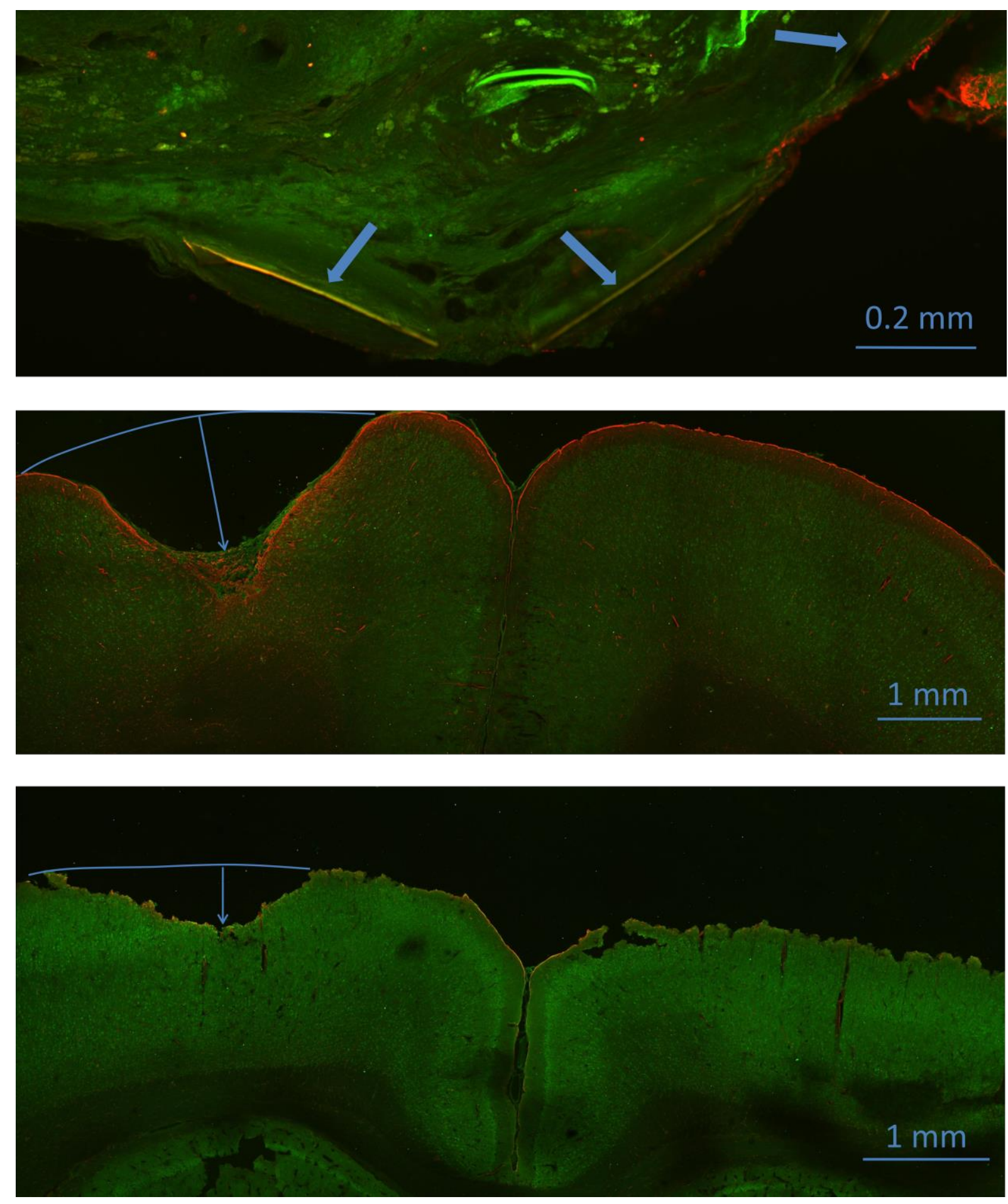

Figure 13: GFAP (red) and NeuN stained slices taken one year after the beginning of the experiment, showing respectively the presence of the electrode in scar tissue (thick arrows indicate $450 \mu \mathrm{m}$ wide polyimide islands that could be retrieved), and the tissue damage seen in MC4 and MC5. Depth of cavity is $1300 \mu \mathrm{m}$ (center) and $450 \mu \mathrm{m}$ (bottom), respectively. 


\section{Conclusion}

The novel concept of micromachined neural implants having a porous resorbable matrix as main constituent was introduced. This concept opens up the possibility of applying materials and techniques from the field of tissue engineering to further improve neural implant technology.

Proof-of-concept electrode arrays were fabricated, demonstrating a fabrication process that allows producing electrode arrays consisting of a down to $5 \mu \mathrm{m}$ thin nonresorbable platinum-polyimide or platinum-Parylene $\mathrm{C}$ backbone reinforced by a resorbable porous chitosan matrix.

In order to improve foldability over three-dimensional curved surfaces such as present in brain, the thin film backbone is finely patterened. The demonstrated process allows insulated freestanding electrical connections down to $10 \mu \mathrm{m}$ wide to be reliably fabricated.

Long term in vivo testing has shown the possibility of using these arrays to record ECoG signals over a period of up to 12 months.

\section{Acknowlegements}

We would like to gratefully acknowledge the FWO Flanders for the postdoctoral fellowship granted to F. Ceyssens and K. van Kuyck. The Laboratory of Experimental Functional Neurosurgery is also supported by the BrainSTAR project of the IWT. Furthermore, we'd like to thank Imec, Belgium, and in particular Wolfgang Eberle and Olga Krylychkina for their help in the histological experiments. Also, special thanks to Jan Fransaer (MTM, KULeuven).

\section{References}

Aarts, A., Neves, H., Puers, R., Van Hoof, C., An interconnect for out-of-plane assembled biomedical probe arrays. Journal of Micromechanics and Microengineering 18, 064004 (2008).

Aarts, A. A. A., Srivannavit, O., Wise, K. D., Yoon, E., Puers, R., Hoof, C. V., Neves, H. P., Fabrication technique of a compressible biocompatible interconnect using a thin film transfer process. Journal of Micromechanics and Microengineering 21 (7), 074012 (2011)

Bai, Q., Wise, K., Anderson, D., A high-yield microassembly structure for three-dimensional microelectrode arrays. Biomedical Engineering, IEEE Transactions on 47 (3), 281289 (2000).

Blanche, T., Spacek, M., Hetke, J., Swindale, N., Polytrodes: high-density silicon electrode arrays for large-scale multiunit recording. Journal of neurophysiology 93 (5), 2987 (2005).

Brandenberg, G., Leibrock, L., Shuman, R., Malette, W., Quigley, H., Chitosan: a new topical hemostatic agent for diffuse capillary bleeding in brain tissue. Neurosurgery 15 (1), 9 (1984) 
Chao, Z., Nagasaka, Y., Fujii, N., Long-term asynchronous decoding of arm motion using electrocorticographic signals in monkeys. Frontiers in neuroengineering 3 (2010).

Chestek, C., Batista, A., Santhanam, G., Yu, B., Afshar, A., Cunningham, J., Gilja, V., Ryu, S., Churchland, M., Shenoy, K., Single-neuron stability during repeated reaching in macaque premotor cortex. The Journal of Neuroscience 27 (40), 10742 (2007)

Crompton, K., Tomas, D., Finkelstein, D., Marr, M., Forsythe, J., Horne, M., Inflammatory response on injection of chitosan/GP to the brain. Journal of Materials Science: Materials in Medicine 17 (7), 633639 (2006)

Cui, X., Lee, V., Raphael, Y., Wiler, J., Hetke, J., Anderson, D., Martin, D., Surface modification of neural recording electrodes with conducting polymer/biomolecule blends. Journal of biomedical materials research 56 (2), $261272(2001)$

Freeman, W., Rogers, L., Holmes, M., Silbergeld, D., Spatial spectral analysis of human electrocorticograms including the alpha and gamma bands. Journal of neuroscience methods95 (2), 111121 (2000)

Haberler, C., Alesch, F., Mazal, P., Pilz, P., Jellinger, K., Pinter, M., Hainfellner, J., Budka, H., 2000. No tissue damage by chronic deep brain stimulation in Parkinson's disease. Annals of neurology 48 (3), 372376 (2000)

Henderson, J., Rodriguez, M., O'Sullivan, D., Pell, M., Fung, V., Benabid, A., Halliday, G., 2004. Partial lesion of thalamic ventral intermediate nucleus after chronic high-frequency stimulation. Movement disorders 19 (6), 709711 (2004)

Ho, M., Kuo, P., Hsieh, H., Hsien, T., Hou, L., Lai, J., Wang, D., Preparation of porous scaffolds by using freezeextraction and freeze-gelation methods. Biomaterials 25 (1), 129138 (2004)

Hosp, J. A., Molina-Luna, K., Hertler, B., Atiemo, C. O., Stett, A., Luft, A. R., Thin-film epidural microelectrode arrays for somatosensory and motor cortex mapping in rat. Journal of Neuroscience Methods 172 (2), 255262 (2008)

Hudak, E.M., Mortimer J.T., Martin H.B., Platinum for neural stimulation: voltammetry considerations. Journal of Neural Engineering 7 (2), 026005 (2010)

Hure, J., Roman, B., Bico, J., Wrapping an adhesive sphere with an elastic sheet. Phys. Rev. Lett. 106, 174301 (2011)

Kim, D., Martin, D., Sustained release of dexamethasone from hydrophilic matrices using PLGA nanoparticles for neural drug delivery. Biomaterials 27 (15), 30313037 (2006)

Kim, D., Viventi, J., Amsden, J., Xiao, J., Vigeland, L., Kim, Y., Blanco, J., Panilaitis, B., Frechette, E., Contreras, D., et al., Dissolvable films of silk fibroin for ultrathin conformal bio-integrated electronics. Nature materials 9 (6), 511517 (2010)

Kim, I., Seo, S., Moon, H., Yoo, M., Park, I., Kim, B., Cho, C., Chitosan and its derivatives for tissue engineering applications. Biotechnology Advances 26 (1), 121 (2008)

Kipke, D., Vetter, R., Williams, J., Hetke, J., Silicon-substrate intracortical microelectrode arrays for long-term recording of neuronal spike activity in cerebral cortex. IEEE Transactions on Neural Systems and Rehabilitation Engineering 11 (2), 151155 (2003)

Lee, K., Ha, W., Park, W., Blood compatibility and biodegradability of partially n-acylated chitosan derivatives. Biomaterials 16 (16), 12111216 (1995) 
Lofti,P., Foreign body reactions to neural implants in the brain. p. 19-22, ProQuest (2007).

Metz, S., Bertsch, A., Renaud, P., Partial release and detachment of microfabricated metal and polymer structures by anodic metal dissolution. Journal of Microelectromechanical Systems 14 (2), 383391 (2005)

Mokwa, W., Technologies for epiretinal stimulation of the retina. Journal of Micromechanics and Microengineering 14(9) (2004)

Mountcastle, V., The columnar organization of the neocortex. Brain 120 (4), 701 (1997)

Musa, S., Welkenhuysen, M., Prodanov, D., Eberle, W., Bartic,C., Nuttin, B.,. Borghs,G., In vitro and in vivo electrochemical characterization of a microfabricated neural probe. Conf Proc IEEE Eng Med Biol Soc. (2009)

Musa,S., Rand, D.R., Bartic,C., Eberle,W., Nuttin, B., Borghs,G., Coulometric detection of irreversible electrochemical reactions ocuuring at Pt microelectrodes used for neural stimulation. Analytical Chemistry $83,4012-4022$ (2011)

Peeters, E., Puers, B., Sansen, W., Gybels, J., De Sutter, P., A two-wire, digital output multichannel microprobe for recording single-unit neural activity. Sensors and Actuators B: Chemical 4 (1-2), 217223 (1991)

Polikov, V., Tresco, P., Reichert, W., Response of brain tissue to chronically implanted neural electrodes. Journal of neuroscience methods 148 (1), 118 (2005)

Rubehn, B., Bosman, C., Oostenveld, R., Fries, P., Stieglitz, T., A MEMS-based flexible multichannel ECoGelectrode array. Journal of neural engineering 6, 036003 (2009)

Ryu, S., Shenoy, K., Human cortical prostheses: lost in translation? Neurosurgical Focus 27 (1), 5 (2009).

Schalk, G., Can electrocorticography (ECoG) support robust and powerful braincomputer interfaces? Frontiers in neuroengineering 3 (2010)

B. K. Sharma, B.K., Electro Chemistry, Krishna Prakashan Media, p. 269 (1997).

Tomihata, K., Ikada, Y., 1997. In vitro and in vivo degradation of films of chitin and its deacetylated derivatives. Biomaterials 18 (7), 567575 (1997)

van Kuyck, K., Welkenhuysen, M., Arckens, L., Sciot, R., Nuttin, B., Histological alterations induced by electrode implantation and electrical stimulation in the human brain: A review. Neuromodulation: Technology at the Neural Interface 10 (3), 244261 (2007).

Viventi, J., Kim, D., Vigeland, L., Frechette, E., Blanco, J., Kim, Y., Avrin, A., Tiruvadi, V., Hwang, S., Vanleer, A., et al., Flexible, foldable, actively multiplexed, high-density electrode array for mapping brain activity in vivo. Nature neuroscience 14,1599-1605 (2011)

Voormolen, J., Ringers, J., Bots, G., van der Heide, A., Hermans, J., Hemostatic agents: Brain tissue reaction and effectiveness: A comparative animal study using collagen fleece and oxidized cellulose. Neurosurgery 20 (5), 702 (1987).

Wedmore, I., McManus, J., Pusateri, A., Holcomb, J., A special report on the chitosan-based hemostatic dressing: experience in current combat operations. The Journal of trauma 60 (3), 655 (2006).

Wise, K., Anderson, D., Hetke, J., Kipke, D., Naja, K., Wireless implantable microsystems: high-density electronic interfaces to the nervous system. Proceedings of the IEEE 92 (1), 7697 (2004) 
Zhong, Y., Bellamkonda, R., Controlled release of anti-inflammatory agent $\alpha$-msh from neural implants. Journal of controlled release 106 (3), 309318 (2005) 